\title{
Design and Experimentation of Automatic Tidying and Sorting Mechanism for Blood Collection Needles in Stacking State
}

\author{
Junhua Tong ${ }^{1,2}{ }^{-}$, Yingpeng Zhu ${ }^{1}$, Leiying $\mathrm{He}^{1}$, Chuanyu $\mathrm{Wu}^{1,2^{*}}$ and Peilin Cheng ${ }^{1}$
}

\begin{abstract}
Disposable blood collection needles become severely intertwined and hooked during stacking, and thus individually feeding disposable blood collection needles during mechanical packaging is difficult. Based on the physical characteristics of the blood collection needles during the stacking state, this study designed an automatic tidying and sorting mechanism by combining compound vibration, sorting, and conveying. During the feeding process, the compound vibration-type material-tidying mechanism tidies 20-30 blood collection needles first; then, the material sorting and conveying mechanism transports the tidied blood collection needles individually. The orthogonal testing of the automatic material tidying process shows that various experimental factors are ranked by the significance level of the effect on the tidying process and the significance ranking is as follows: vertical vibration frequency > horizontal amplitude > vertical amplitude > horizontal vibration frequency. Experiments were performed after analyzing the optimal combination. The results demonstrate that when the horizontal vibration frequency is $1.7 \mathrm{~Hz}$, the horizontal amplitude is $150 \mathrm{~mm}$, vertical vibration frequency is $1.3 \mathrm{~Hz}$, vertical amplitude is $30 \mathrm{~mm}$, and material length after tidying is $265 \mathrm{~mm}$. The automatic sorting and conveying experiment shows the effect of various experimental factors on the feed rate of the material, where the significance level of the effect is ranked as follows: vibration frequency $>$ material quantity > channel dip angle. The experimental results show that when the number of materials is 25 , the channel dip angle is $12^{\circ}$, and vibration frequency is $52.5 \mathrm{~Hz}$. The material delivery efficiency reaches $0.51 \mathrm{~s} /$ root, meeting the requirement of five channels for 80000 root/day feeding efficiency. The study can provide reference for the realization of automatic feeding of large aspect ratio flexible materials in similar stacking state.
\end{abstract}

Keywords: Packaging, Automatic feeding, Stacking state, Automatic tidying, Sorting and conveying

\section{Introduction}

In recent years, automation in packaging machineries has become increasingly important. Many industries have intensified their dependence on it and they need to continuously improve and innovate [1-3]. Automatic feeding as the primary link of automatic packaging is a key factor in achieving automatic packaging, and this technology is also the focus of research in automatic packaging machinery [4-6].

\footnotetext{
*Correspondence: cywu@zstu.edu.cn

1 Faculty of Machinery and Automation, Zhejiang Sci-Tech University, Hangzhou 310018, China

Full list of author information is available at the end of the article
}

According to the development of automatic feeding technology and the demand for product packaging techniques at local and international markets, the research on automatic feeding technology mainly focuses on four aspects: modularization, intelligentization, serialization, and standardization [7-9]. Automatic feeding significantly increases packaging efficiency and reduces packaging costs compared to manual feeding and semiautomatic feeding [10-12]. At present, there are many studies on the grasping manipulator, locally and internationally, focusing mainly on structural design and control methods [13-15]. These manipulators use electric, fluid, and pneumatic systems to drive the manipulator joints to complete the grip function [16-18], and the structure is relatively complex. For grasping small volume materials, 
it is often necessary to link the sensor detection technology. The response time of a manipulator is long, and it is difficult to meet the requirements of material distribution. Ramalingam et al. [19] analyzed the characteristics of the small parts of a linear vibratory feeder. They designed a roller-type channel with radial fins to send components into the feeding track, where the track shape can be altered to convey the different components. They also established a mathematical model to calculate the conveying speed. Loy et al. [20] focused on the problem of modules having more replacements and higher feed costs because of the different materials in vibratory hopper feeds; then, they designed a new modular vibratory hopper to completely separate the product-oriented module from the hopper and improve applicability of the vibration channel and reduce cost of feeding. Wang et al. [21], according to the principle of the vibrating feeder, proposed a method to achieve directional transportation for corn seeds. They explored the relationship between the various postures of the change process and mechanical parameters during orientation. Meng et al. [22] proceeded from pose modelling of connecting rod kinematics to using inverse kinematics analysis method and dimension vector chain method to compensate the error in the joints of a parallel-type curve feeding platform and corrected the feed deviation of this mechanism when feeding the band saw machine joiner. Xu et al. [23], designed an automatic manipulator based on mechanical and electrical integration and control technology, which could simulate artificial operation and achieve pickup, measurement, and filling for filamentous materials. Chandravanshi et al. [24] used a vibratory feeder as a single-degree-of-freedom forced-vibration system, established its mathematical model, conducted a dynamic analysis to obtain the influence of the working frequency of the vibrating feeder on the material conveying rate, and administered experimental verification. Xing et al. [25] studied the dissemination and sequencing transport mechanism of stacked maize seeds in an electromagnetic vibration hopper through theoretical analysis, EDEM simulation and practical experiments. The test results demonstrated that when the diameter and height of the hopper were determined, the factors affecting the movement state and efficiency of the maize seeds' dispersion and arraying transport, in the electromagnetic vibration hopper, were the bottom inclination angle, track screw pitch, and working voltage. Jiang et al. [26] applied a linear piezoelectric actuator, driving circuit, and position sensor to an electron microscope sampling container and designed an automatic feeding system that uses capillary action to sample the microfluidic behavior. Nevertheless, the feeding objects of the above automatic feeding devices were mostly rigid materials. Although there are numerous studies on automatic feeding devices globally, they are not applicable to flexible materials that are easily deformed.

At present, there are only few studies on flexible material automatic feeding devices. One such study was presented by Koo et al. [27]. They used object modelling technology to systematically analyze the paper-feeding mechanism design of a printing press, hierarchically designed each part of the paper-feeding mechanism, and converted the design results into a bill of materials data for production, thereby improving the operating speed of the printing press. Han et al. [28] aimed at the problem of low manual feeding efficiency for basket-type transplanters in the Xinjiang Province, and designed an auto-feeding system of plug seedlings transplanted with lateral and longitudinal displacements. The system was controlled by a PLC, and the results verified the reliability of the system with respect to transporting the seedlings. Tong et al. [29] considered cucumber seedlings as their research object, with the pointer clamping angle, number of gripping fingers, moisture content of the nursery, growth of the three groups of seedlings, and volume ratio of the two nursery substrates as the influencing factors. By considering the clamping force of the pointer to the nursery as the optimization target, the analysis of the single influencing factor was carried out. The test results demonstrated that all five factors had an effect on the change of clamping force; the optimum value for each factor was: pointer clamping angle at $7^{\circ}$, the number of gripping fingers was four fingers, the moisture content level of the nursery was $84 \%$, growth of the seedlings was medium growth and above, and the ratio of matrix volume was 6:3:1. Tong et al. [30] designed an automatic transportation and replantation device suitable for a double-root grafting machine to solve the problem of the grafted seedlings relying on manual transport and replanting operation; subsequently, they conducted simulation and kinematic analysis on the key mechanism and determined the optimal conditions for the device through an orthogonal test. Doyo et al. [31] invented a self-locking paper feeding mechanism to achieve a continuous supply of fixed length papers. Zhao et al. [32] focused on the planting patterns of field planting in Xinjiang, then used a timing belt and guide channel mechanism to design an automatic feed mechanism for a transplanter. They used PLC to control feed distance to achieve the automatic feeding process in the transplanter.

The research object of this paper is blood collection needles, which are flexible, exhibit easy accumulation, and have large length-to-diameter ratios. The existing feeding method is difficult to use in the single separation and delivery process of mechanical packaging. Therefore, this paper investigated an automatic feeding method, where tidying is performed first, then sorting, 
and conveying. An automatic tidying and sorting mechanism that combines compound vibration, sorting, and conveying was designed. The key structural and operating parameters of the compound vibration-type material-tidying and material sorting, and conveying mechanisms were determined through automatic material tidying and sorting experiments. These experiments provided a technical reference for the development of

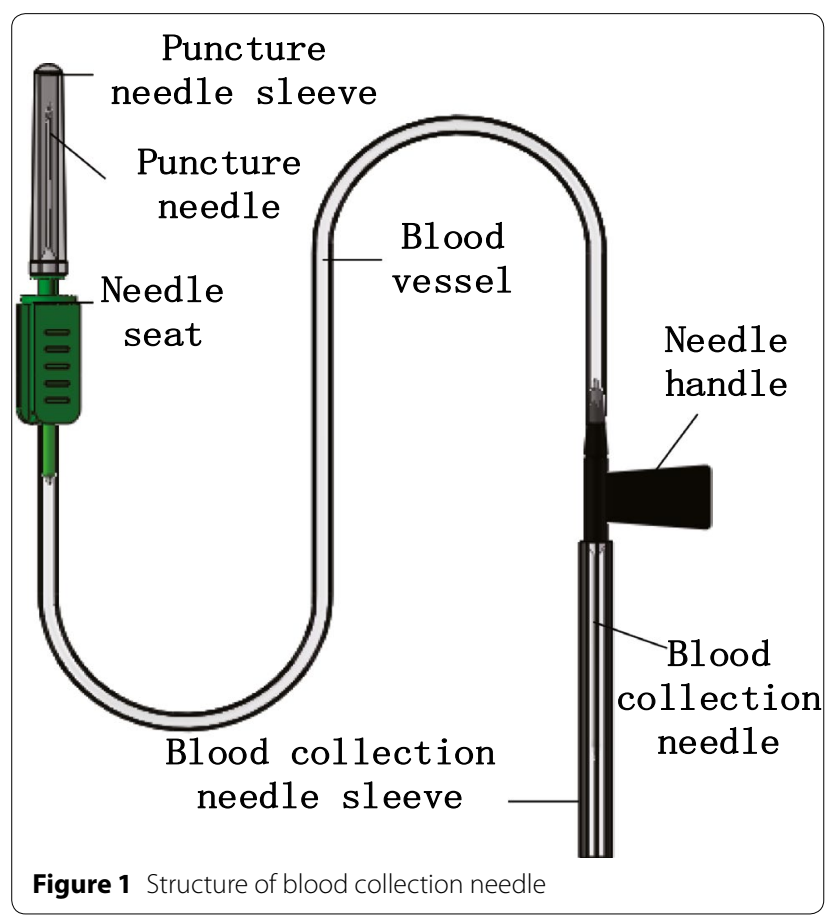

the automatic packaging device for flexible blood collection needles in the stacking state.

\section{Analyses of the Physical Characteristics of Blood Collection Needles and General Scheme for Automatic Feeding}

The blood collection needle is a plastic hose. Its material is soft, and its geometric shape is uncertain, as shown in Figure 1.

The statistical measurement of blood collection needle weight $(m)$ is $2.2 \mathrm{~g}$, its needle diameter $(d)$ is $2 \mathrm{~mm}$, and its length $(L)$ is $263 \mathrm{~mm}$. the internal arrangement of the blood collection needles in the stacking state is scattered and the length of the material in its stacking state is shorter than its natural state. Therefore, the measured length of the blood collection needles in the stacking state is $260 \mathrm{~mm}$, as shown in Figure 2.

The automatic feeding of blood collection needles in the stacking state was achieved by adopting an automatic feeding method wherein the sorting process was performed after the tidying process. The prototype tested the effect of material tidying and material sorting. The

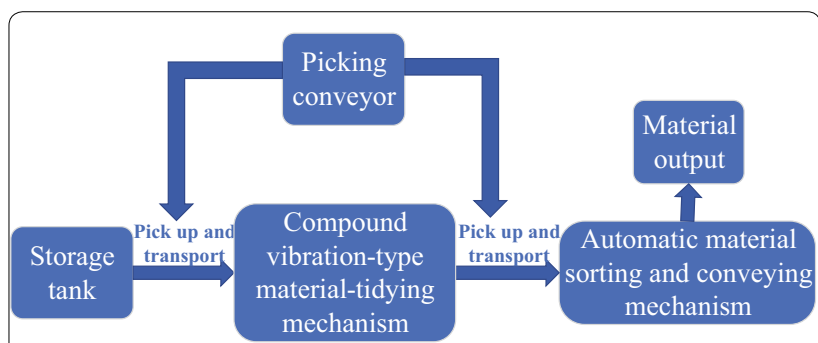

Figure 3 Overall module layout of automatic feeding device

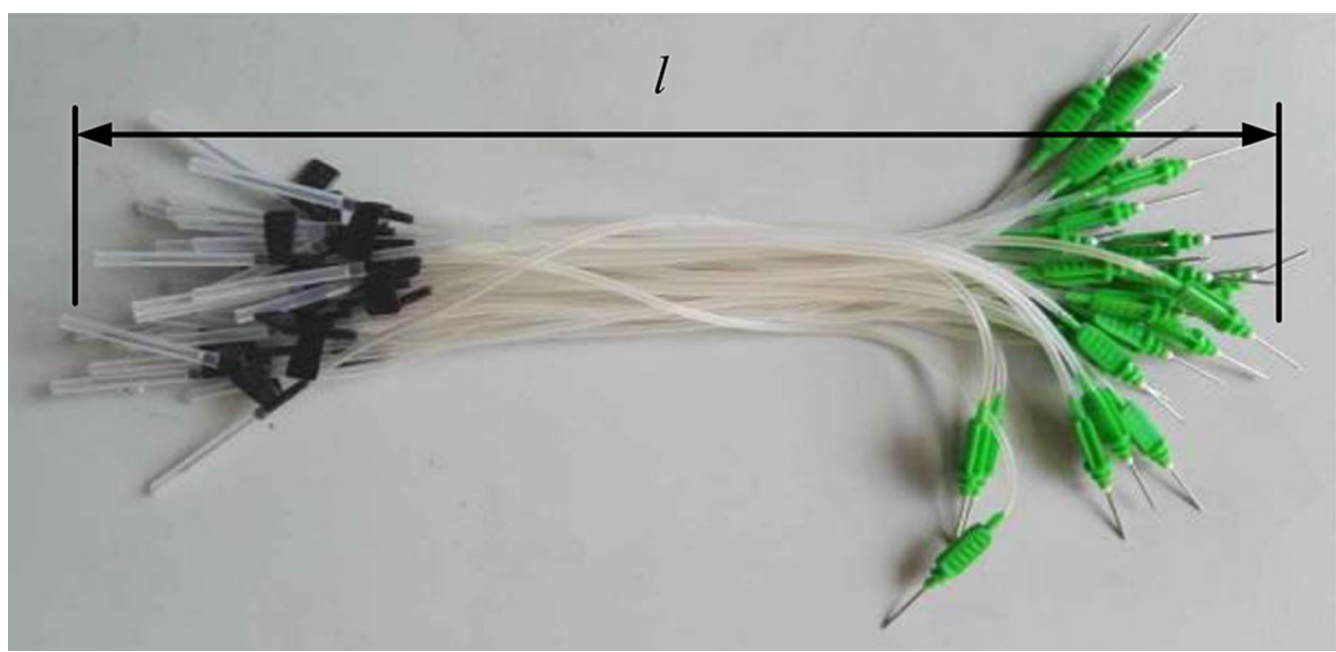

Figure 2 Length measurement of blood collection needles in the stacking state 
working process is shown in Figure 3, where the picking conveyor drives the pick-up mechanism to grasp the quantitative and scattered medical venous blood collection needles and deposits them into the compound vibration-type material-tidying mechanism. After the materials are tidied, they are transported to the automatic material sorting and conveying mechanism through the picking conveyor for sorting. Finally, a single blood collecting needle is grabbed by the external pick-up unit and placed into the feeding channel of the packing device. The compound vibration-type material-tidying mechanism and the automatic material sorting and conveying mechanism are the core of the automatic tidying and sorting mechanism, and the picking conveyor is an auxiliary module. The design of the two mechanisms in the automatic tidying and sorting process will be discussed in subsequent sections, and the effects of material tidying, and material sorting are tested by the prototype.

\section{Compound Vibration-Type Material-Tidying Mechanism Design}

By combining the various studies on the interactions of horizontal and vertical vibration sorting and picking of scattered materials, a compound vibration-type material-tidying mechanism for blood collection needles is designed, as shown in Figure 4. The horizontal vibration transmission mechanism is a centered crank slider mechanism, and the driving source is a motor. The horizontal vibration is achieved by reciprocating the motion of the slider. Vibration frequency is depicted by the motor rotation frequency, and amplitude is the slide stroke. The cylinder is the driving source for vertical vibration and it is achieved by controlling the reciprocating expansion of the cylinder piston rod. The vibration frequency is the telescopic frequency of the cylinder piston rod with the amplitude as the cylinder stroke. The scattered materials are placed in the material frame and removed by the robot through the assistance of a picking-aided mechanism after the tidying process. The material frame and reclaiming auxiliary mechanism are fixed at the end of the cylinder piston rod determined by the vertical vibration mechanism. The material frame and vertical vibration mechanism are fixed in the slider of the horizontal vibration mechanism. The process for tidying the materials of the compound vibration-type material-tidying mechanism is divided into four actions, as shown in Figure 5 . Figure $5(\mathrm{a})$ and $5(\mathrm{c})$ show that materials are aligned in the horizontal direction by the impact between the materials and the two side walls of the material frame because of the effect of horizontal vibration when the mechanism is working. The material is thrown up and falls back to the bottom of the material frame because of the vertical vibration action. This ensures that the hooking and winding among the materials are gradually weakened, as shown in Figure 5(b) and 5(d). The scattered blood collection needles are aligned under the cyclic activity of the four actions shown in Figure 5. According to the analysis of the movement of the mechanism, horizontal vibration frequency, horizontal amplitude, vertical vibration frequency, vertical amplitude, length of the material frame, and single material consumption time are all factors that have an influence on the effect of tidying the materials.

The length $(l)$ of the blood collection needle in its natural state is $260 \mathrm{~mm}$, and the material frame length $(D)$ is greater than $l$. To determine the material frame length $(D)$ and single material consumption time $\left(T_{1}\right)$, this study selected $270,290,300,320,340,360,380$, and $400 \mathrm{~mm}$ as the different values of material frame length $(D)$. Then, we installed the different lengths of material frames on the compound vibration-type material-tidying mechanism for experimentally exploring

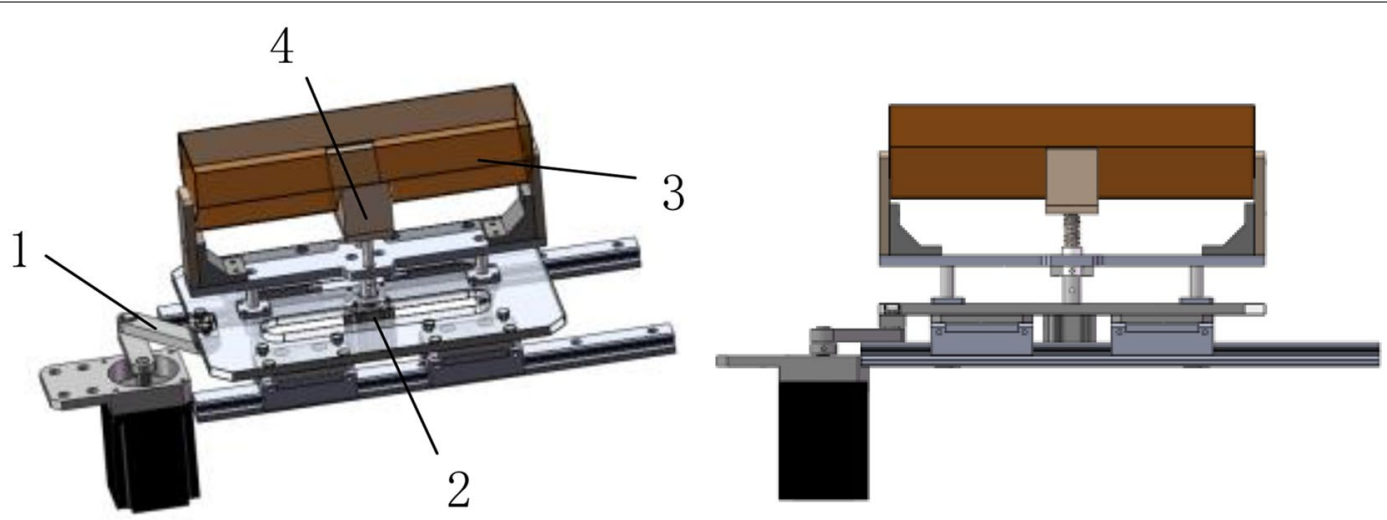

Figure 4 Compound vibration-type material-tidying mechanism diagram. 1. Crank slider mechanism; 2. Cylinder; 3. Material frame; 4. Reclaiming auxiliary mechanism 


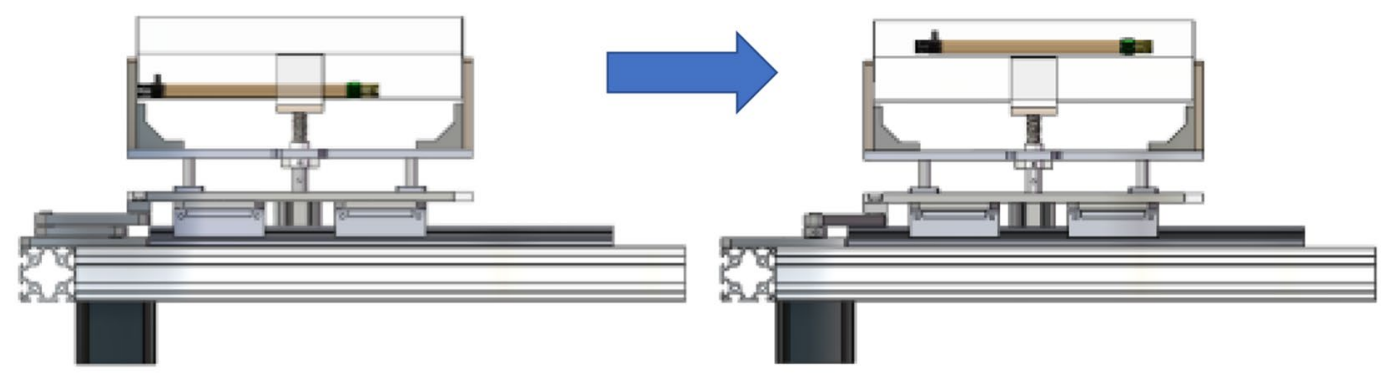

$\mathbf{a}$

b

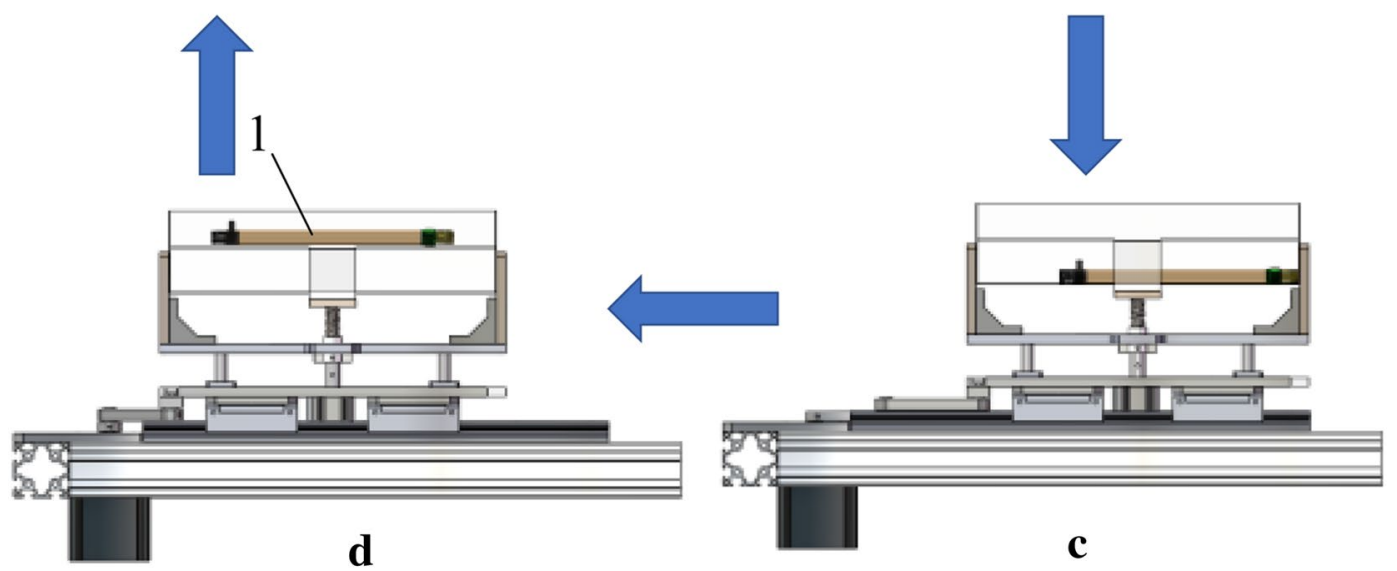

Figure 5 Compound vibration process diagram: a touch bottom stage one; $\mathbf{b}$ throw up stage one; $\mathbf{c}$ touch bottom stage two; $\mathbf{d}$ throw up stage two. 1. Blood collection needles

the effects of the tidying process. The test results showed that the material frame with length $\leq 340 \mathrm{~mm}$ was too short, and the frequency of impact between the two ends of the material and both sides of the material frame was large, which resulted in the material easily jumping out of the material frame. The material frame with length $\geq 360 \mathrm{~mm}$ was too large, thereby resulting in a lesser likelihood of a collision between both sides of the material and the material frame. Thus, the material tidying process was deteriorated. Therefore, considering the middle value, the material frame length $D$ was fixed at $350 \mathrm{~mm}$ to ensure that the material does not jump out of the material frame and display a good tidying effect. The tidying material effect exploration experiment was conducted with the selected material frame, and the time from the start of the tidying process till the material had stabilized was approximately $40 \mathrm{~s}$, with the single material time $T_{1}$ selected to be $40 \mathrm{~s}$.

After $D$ and $T_{1}$ were determined, the values of the horizontal vibration frequency, horizontal amplitude, vertical vibration frequency, and vertical amplitude were determined according to the results of the subsequent orthogonal test analysis.

\section{Orthogonal Test Design and Analysis of Automated Material Tidying Experiment}

\subsection{Evaluation Index of Experiment}

Hooking and winding between the blood collection needles is minimal after the material is tidied. The parameter that reflects the uniformity of the materials is the total length of the collection of blood needles in the material frame, as shown in Figure 6. The total length $\left(S_{2}\right)$ of the material after tidying can be used as an evaluation index of the uniformity of materials in the test.

\subsection{Experimental Design}

To determine the range of the horizontal vibration frequency $f_{1}$, horizontal amplitude $L_{1}$, vertical vibration frequency $f_{2}$, and vertical amplitude $L_{2}$ in the experiment, the automatic tidying testing platform shown in Figure 7 was setup for the exploration of the tidying effect of the stacked and scattered blood collection needles. According to the exploration experiment, when the tidying time was more than $50 \mathrm{~s}$, there was no obvious change in the tidying effect. Therefore, the tidying time of each material tidying cycle was set to a maximum time of $50 \mathrm{~s}$. Test results showed that the 


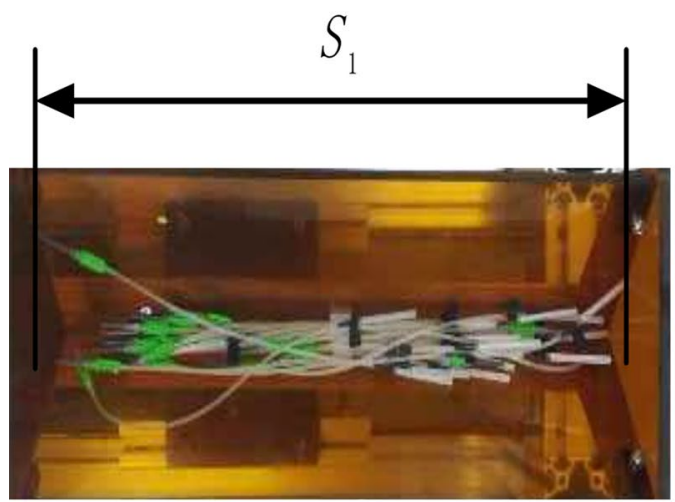

$\mathbf{a}$

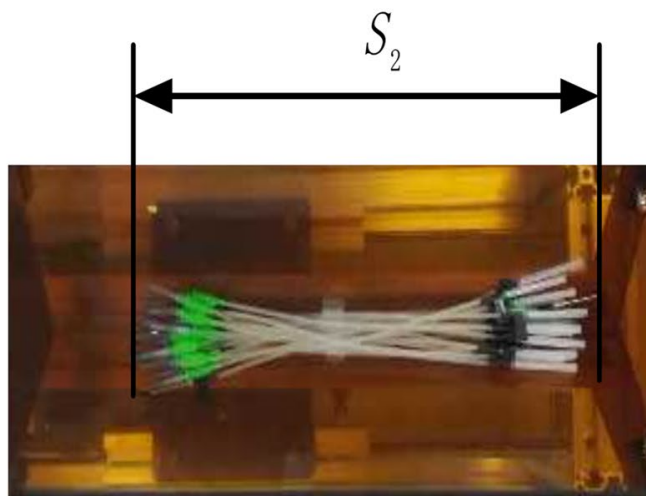

b

Figure $\mathbf{6}$ Total length of the blood collection needles in the material frame: a materials before tidying process; $\mathbf{b}$ materials after tidying process

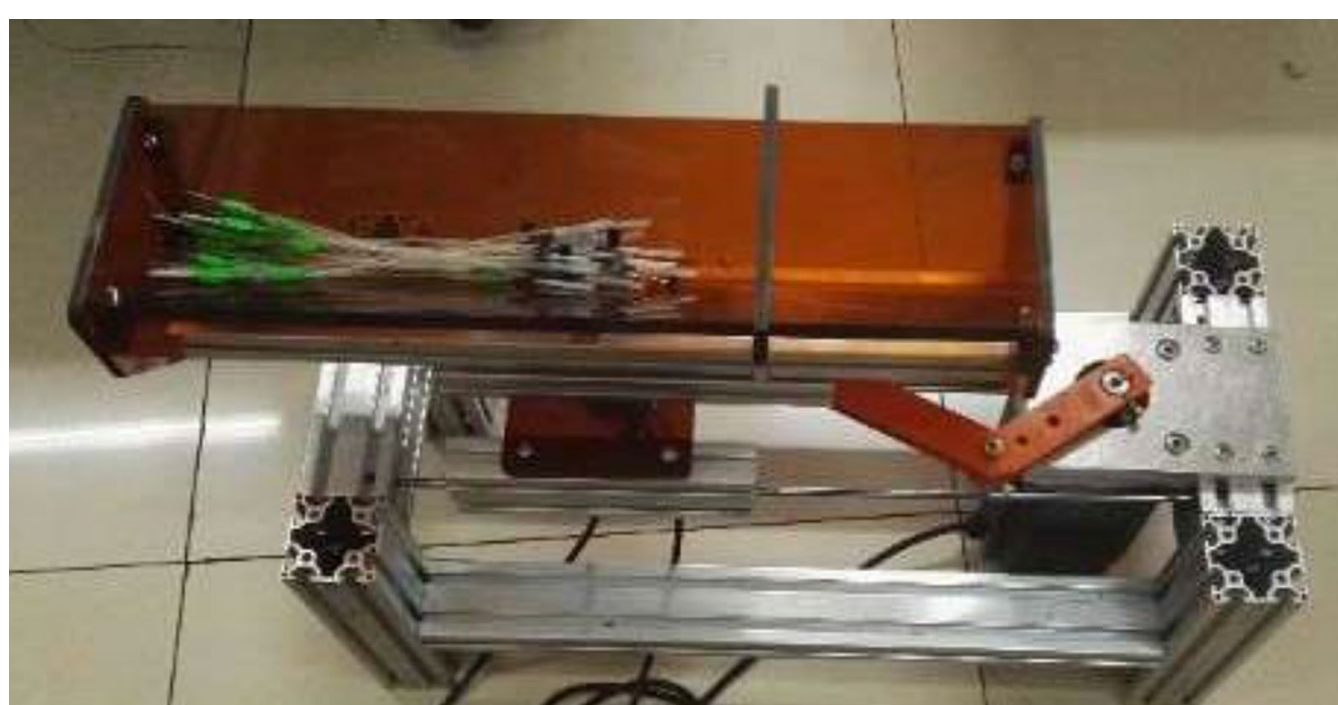

Figure 7 Automatic material tidying test bench

values of $f_{1}$ and $L_{1}$ affected the material tidying effect in the horizontal direction. For smaller values of $f_{1}$ and $L_{1}$, it was considerably unlikely for the material to collide with either side of the material frame. A minimum value of $1.5 \mathrm{~Hz}$ for $f_{1}$ and $100 \mathrm{~mm}$ for $L_{1}$ was required to achieve optimum collision. Conversely, for larger values of $f_{1}$ and $L_{1}$, the two ends of the material were more likely to collide with either side of the material frame, which may easily cause the material to jump out of the material frame. To avoid this, the maximum value of $f_{1}$ and $L_{1}$ were limited to $1.7 \mathrm{~Hz}$ and $200 \mathrm{~mm}$, respectively.

We determined that the values of $f_{2}$ and $L_{2}$ have significant influence on eliminating the phenomenon of hooking and winding between materials. It was identified that for smaller values of $f_{2}$ and $L_{2}$, a lesser number of times the materials were thrown and the throwing height in each cycle was lower. Therefore, it was more challenging to eliminate the hooking and winding between materials. Consequently, the minimum values of $f_{2}$ and $L_{2}$ should be at least $1.3 \mathrm{~Hz}$ and $20 \mathrm{~mm}$, respectively. Conversely, for larger values of $f_{2}$ and $L_{2}$ the materials became more scattered and it was easier for the materials to jump out of the material frame. Therefore, the maximum values of $f_{2}$ and $L_{2}$ were limited to $2.5 \mathrm{~Hz}$ and $30 \mathrm{~mm}$, respectively. The test levels of $f_{1}, L_{1}, f_{2}$, and $L_{2}$ are shown in Table 1 .

The orthogonal test of automatic material tidying was administered by using $\mathrm{L}_{9}\left(3^{4}\right)$ orthogonal array arrangement. Ten tests were performed for each combination of test conditions, and the average value $\bar{S}_{2}$ of the total 
length $S_{2}$ of the material after ten tests was considered as the result for each set of tests.

\subsection{Results and Analysis}

The range analysis was used on the test results shown in Table 2. The order of the factors affecting the total length of the materials after tidying is as follows: vertical vibration frequency, horizontal amplitude, vertical amplitude, and horizontal vibration frequency. The optimal level combination includes the following conditions: horizontal vibration frequency $f_{1}$ of $1.7 \mathrm{~Hz}$, horizontal amplitude $L_{1}$ of $150 \mathrm{~mm}$, vertical vibration frequency $f_{2}$ of $1.3 \mathrm{~Hz}$, vertical amplitude $L_{2}$ of $30 \mathrm{~mm}$, and total length of the material piled after tidying was $265 \mathrm{~mm}$.

This study determined after the experiment that the vertical vibration frequency had the most significant effect on the total length of the material after tidying. According to the column of vertical vibration frequency in Table $2, k_{1}<k_{2}$ and $k_{2}>k_{3}$, which shows that the total length of the material increases first, then decreases with the increasing vertical vibration frequency. With the

Table 1 Automated material tidying orthogonal test factors and levels

\begin{tabular}{|c|c|c|c|c|}
\hline \multirow[t]{2}{*}{ Level } & \multicolumn{4}{|l|}{ Factor } \\
\hline & $\begin{array}{l}\text { Horizontal } \\
\text { vibration } \\
\text { frequency } \\
f_{1}(\mathrm{~Hz})\end{array}$ & $\begin{array}{l}\text { Horizontal } \\
\text { amplitude } \\
L_{1}(\mathrm{~mm})\end{array}$ & $\begin{array}{l}\text { Vertical } \\
\text { vibration } \\
\text { frequency } \\
f_{2}(\mathrm{~Hz})\end{array}$ & $\begin{array}{l}\text { Vertical } \\
\text { amplitude } \\
L_{2}(\mathrm{~mm})\end{array}$ \\
\hline 1 & 1.5 & 100 & 1.3 & 20 \\
\hline 2 & 1.6 & 150 & 1.9 & 25 \\
\hline 3 & 1.7 & 200 & 2.5 & 30 \\
\hline
\end{tabular}

increase in the frequency of vertical vibration, the number of times that the blood collection needles are thrown increases as well. This increase makes the materials increasingly scattered. The hooking between the materials is not significantly declined at this time and results in an inferior tidying effect. However, as the frequency of vertical vibration continues to increase, the hooking and winding between materials starts to weaken considerably, consequently, improving the tidying process.

The effect of horizontal amplitude on the total length of materials had the second significant effect after tidying. Table 2 compares the total length of the materials with horizontal amplitude. The total material length when the horizontal amplitude was $100 \mathrm{~mm}$ was greater than the total material lengths when the horizontal vibrations were $150 \mathrm{~mm}$ and $200 \mathrm{~mm}$. The main reason is that when the horizontal amplitude is smaller, the two ends of the material collide with the ends of the material frame more forcefully, at the same horizontal vibration frequency. Consequently, the material tends to jump out of the material frame, thereby causing a greater disorder of the material after the tidying process.

The horizontal vibration frequency and vertical amplitude have a lesser degree of influence on the total length of the material after the tidying process. In the vertical amplitude range $R$ listed in Table 2, the influence of the two factors on the test results are approximately the same. The reason is that the horizontal vibration frequency mainly affects the collision effect between the material and the material frame during the tidying process. The horizontal vibration frequency changes over a smaller range; therefore, the effects on tidying are minimal, making it an auxiliary factor. The vertical amplitude

Table 2 Orthogonal test results of automatic material tidying process

\begin{tabular}{|c|c|c|c|c|c|}
\hline \multirow[t]{2}{*}{ Test number } & \multicolumn{4}{|l|}{ Factors } & \multirow{2}{*}{$\begin{array}{l}\text { Material } \\
\text { stacking } \\
\text { length }\end{array}$} \\
\hline & $\begin{array}{l}\text { Horizontal vibration } \\
\text { frequency }\end{array}$ & Horizontal amplitude & $\begin{array}{l}\text { Vertical vibration } \\
\text { frequency }\end{array}$ & Vertical amplitude & \\
\hline 1 & 1 & 1 & 1 & 1 & 270 \\
\hline 2 & 1 & 2 & 2 & 2 & 311 \\
\hline 3 & 1 & 3 & 3 & 3 & 270 \\
\hline 4 & 2 & 1 & 2 & 3 & 335 \\
\hline 5 & 2 & 2 & 3 & 1 & 279 \\
\hline 6 & 2 & 3 & 1 & 2 & 273 \\
\hline 7 & 3 & 1 & 3 & 2 & 298 \\
\hline 8 & 3 & 2 & 1 & 3 & 265 \\
\hline 9 & 3 & 3 & 2 & 1 & 294 \\
\hline$k_{1}$ & 283.7 & 301 & 269.3 & 281 & \\
\hline$k_{2}$ & 295.7 & 285 & 313.3 & 294 & \\
\hline$k_{3}$ & 285.7 & 279 & 282.3 & 290 & \\
\hline$R$ & 12 & 22 & 44 & 13 & \\
\hline
\end{tabular}


changes the height to which the material is thrown up; this is mainly to weaken the phenomena of hooking and winding between the blood collection needles. It is also determined as an auxiliary factor in the course of the test.

\section{Automatic Material Sorting and Conveying Mechanism Design}

After the compound vibration-type material-tidying mechanism was used for tidying the blood collection needles while stacking, it needs to be separated and transported by a single root to achieve the automatic feeding function. Combined with the electromagnetic linear vibration feeder under the half-wave rectification power supply, the armature and electromagnet have the function of dividing and conveying the needles. The material sorting and conveying mechanism, with the feeder as the core component, is illustrated in Figure 8. Combined with the geometric physical characteristics of the blood collection needle, the channel is designed as shown in Figure 8. The channel is fixed above the electromagnetic linear vibration feeder, and the channel plane is at an angle $\alpha$ to the horizontal plane.

Under the action of the feeder vibration, the materials were separated and transported in an orderly manner. Based on the different states of the material moving on the material channel, the material separation and transportation process is divided into four stages, as shown in Figure 9. When the mechanism is working, the action of the vibration of the electromagnetic linear vibration feeder causes the material to enter the material channel by the ladder assembly; then the shock generated in the sorting and conveying process ensures the needle seats and ladder assembly fit together, the material with the needle handle facing upwards falls off the material channel, as shown in Figure 9(a) and 9(b). After the materials reach the material barrier with a small width at the end of the material channel, the material is blocked behind the material barrier and is discharged in a single row in the material channel, as shown in Figure 9(c) and 9(d). Subsequently, it is easy for the reclaiming actuator (not shown) to pick up the material continuously at the material barrier port to complete the single separation and conveying function of the material.

According to the results of the analysis on the feeding principle and material sorting process of the vibrating feeder, the vibration frequency, material channel dip angle, and the amount of material affect the efficiency of the material delivery.

\section{Orthogonal Test and Analysis of Automatic Material Sorting and Conveying Experiment}

\subsection{Evaluation Index of Experiment}

The amount of material to be sorted within each working cycle of the material sorting and conveying mechanism is constant once the amount of material is determined. Therefore, the material feeding rate is the most direct parameter for reflecting the sorting efficiency. The method of sorting efficiency can be represented by the number $B$ of materials to be sorted and conveyed per minute, and the time $T$ required for sorting and conveying one blood collection needle. The latter is selected as the target parameter for automatic sorting for ease of operation.

\subsection{Experimental Design}

To determine the range of material quantity $A$, channel dip angle $\alpha$, and vibration frequency $f$ in the experiment, the automatic material sorting and conveying test platform as illustrated in Figure 10 was setup for exploring the effect of sorting. The material entering the automatic
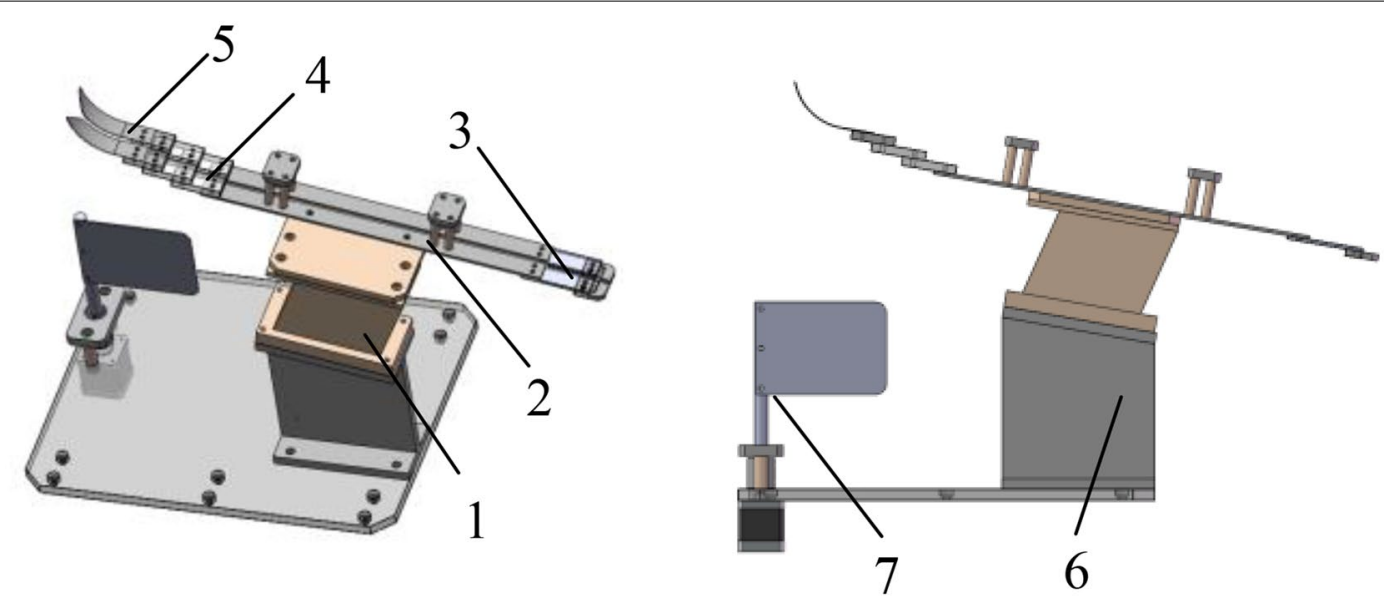

Figure 8 Automatic sorting and conveying mechanism diagram. 1. Electromagnetic linear vibration feeder; 2. Channel; 3. Blocking component; 4. Ladder assembly; 5 . Channel mouth; 6 . Support; 7 . Swing plate 

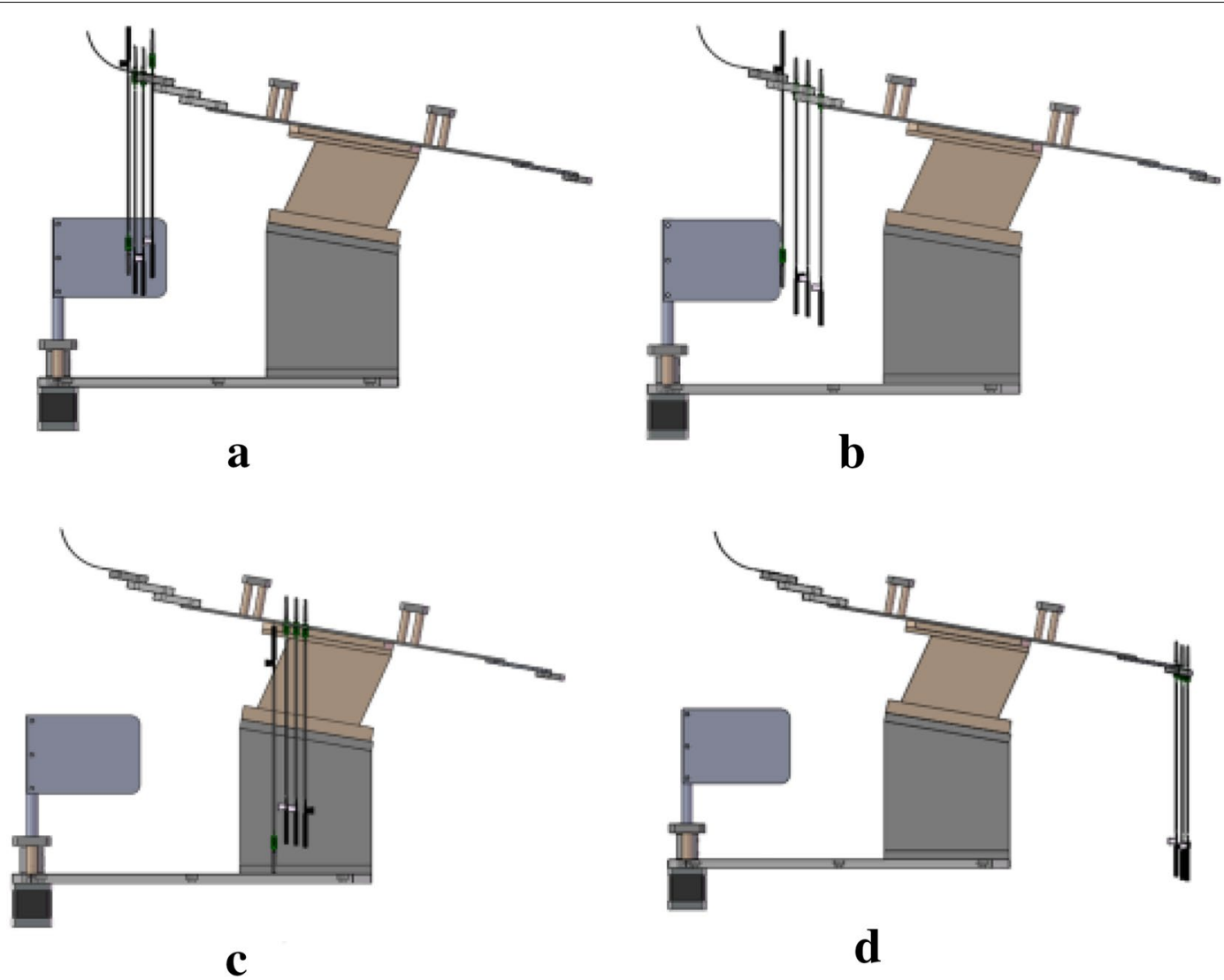

Figure 9 Automatic sorting and conveying process diagram: a entry stage; $\mathbf{b}$ unwinding stage; $\mathbf{c}$ dropping stage; $\mathbf{d}$ recycle stage

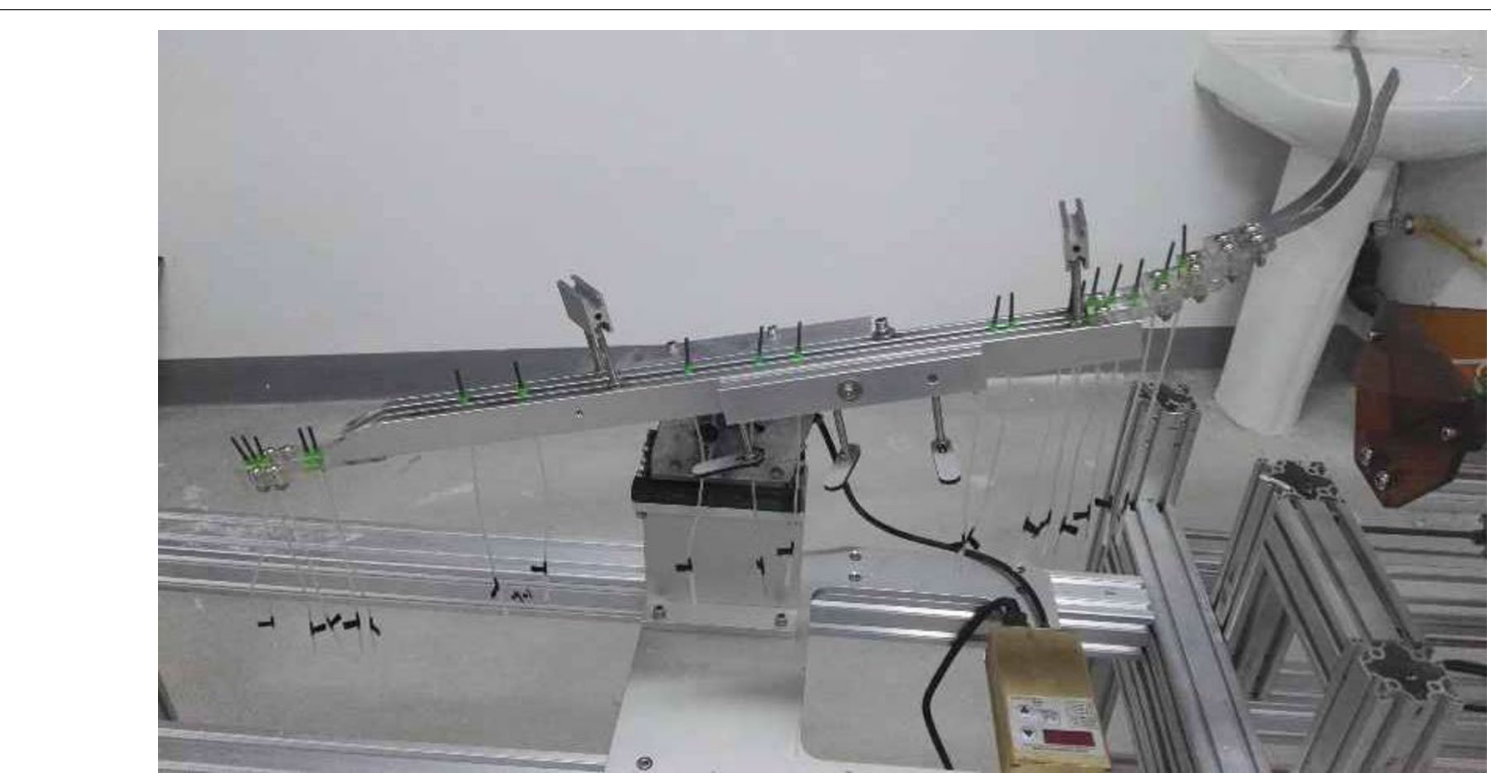

Figure 10 Automatic sorting and conveying test bench 
material sorting and conveying mechanism came from the same compound vibration-type material-tidying mechanism to ensure that the material stacking conditions were similar in each experiment.

On the premise of guaranteeing that the material can smoothly enter the channel and slide on the channel, the test results showed that the value of $A$ directly affected the material sorting effect. The material quantity $A$ affected the time of material entry into the material channel. The minimum value of $A$ was determined to be 20 with a maximum value of 30 to ensure efficiency and guarantee that the material was not blocked at the material channel mouth. The larger the materials channel dip angle $\alpha$, the faster the material falling speed. At the same time, if the channel dip angle $\alpha$ was too large it would influence the vibration effect and reduce the efficiency of material sorting. Therefore, to ensure material sorting efficiency, the minimum required value of the material channel dip angle $\alpha$ was $9^{\circ}$. The material channel dip angle $\alpha$ should be limited to a maximum angle of $15^{\circ}$ to prevent the substantial weakening of the vibration effect, which may lead to the lowering of the material sorting speed. For larger values of vibration frequency $f$, it was more likely that the material would bounce. Thus, intertwining and hooking could occur. If $f$ was extremely small, it would make it difficult for the material to achieve the vibration sorting effect. Therefore, the minimum value of $f$ was $51.5 \mathrm{~Hz}$. A maximum value of $52.5 \mathrm{~Hz}$ was required for reducing the bounce. Therefore, the numerical range for various factors are predetermined as listed in Table 3.

The three experimental factors have a small value range in Table 3. Therefore, the three experimental factors $A, \alpha$, and $f$ are unified into three levels as shown in Table 4.

The material quantity to be fed within each working cycle of the material sorting and conveying mechanism remains constant after the material quantity is determined. Therefore, the material feeding rate is the parameter with the most direct influence on the sorting and conveying efficiency.

An orthogonal test for automatic material sorting and conveying was conducted by the using $L_{9}\left(3^{4}\right)$ orthogonal table array and performing 10 tests for each combination of test conditions. The average time $\bar{T}$ of time $T$ of the

Table 3 Numerical interval of auto-sorting orthogonal test factor

\begin{tabular}{llll}
\hline $\begin{array}{l}\text { Experimental } \\
\text { factors }\end{array}$ & $\begin{array}{l}\text { Material quantity } \\
\boldsymbol{A} \text { (Root) }\end{array}$ & $\begin{array}{l}\text { Channel dip } \\
\text { angle } \\
\boldsymbol{a}\left({ }^{\circ}\right)\end{array}$ & $\begin{array}{l}\text { Vibration } \\
\text { frequency } \\
\boldsymbol{f}(\mathbf{H z})\end{array}$ \\
\hline Numerical range & $20-30$ & $9-15$ & $51.5-52.5$ \\
\hline
\end{tabular}

Table 4 Automatic sorting orthogonal test factors and levels

\begin{tabular}{llll}
\hline Factor level & \multicolumn{3}{l}{ Experimental factors } \\
\cline { 2 - 4 } & $\begin{array}{l}\text { Material } \\
\text { quantity } \\
\boldsymbol{A} \text { (Root) }\end{array}$ & $\begin{array}{l}\text { Channel dip } \\
\text { angle } \\
\boldsymbol{a}\left(\mathbf{(}^{\circ}\right)\end{array}$ & $\begin{array}{l}\text { Vibration } \\
\text { frequency } \\
\boldsymbol{f ( H z )}\end{array}$ \\
\hline 1 & 20 & 10 & 51.5 \\
2 & 25 & 12 & 52.0 \\
3 & 30 & 14 & 52.5 \\
\hline
\end{tabular}

Table 5 Automatic material sorting orthogonal test results

\begin{tabular}{lllll}
\hline Test number & $\begin{array}{l}\text { Factors } \\
\text { Material }\end{array}$ & $\begin{array}{l}\text { Channel } \\
\text { dip angle }\end{array}$ & $\begin{array}{l}\text { Vibration } \\
\text { frequency }\end{array}$ & \\
\cline { 2 - 4 } & quantity & Sorting time \\
1 & 1 & 1 & 1 & 0.77 \\
2 & 1 & 2 & 2 & 1.85 \\
3 & 1 & 3 & 3 & 0.65 \\
4 & 2 & 1 & 2 & 1.57 \\
5 & 2 & 2 & 3 & 0.51 \\
6 & 2 & 3 & 1 & 1.12 \\
7 & 3 & 1 & 3 & 1.55 \\
8 & 3 & 2 & 1 & 1.65 \\
9 & 3 & 3 & 2 & 1.23 \\
$k_{1}$ & 1.09 & 1.30 & 1.18 & \\
$k_{2}$ & 1.07 & 1.34 & 1.55 & \\
$k_{3}$ & 1.48 & 1.00 & 0.90 & \\
$R$ & 0.41 & 0.34 & 0.65 & \\
\hline
\end{tabular}

material sorting and conveying process after 10 tests was considered as the result of each set of tests.

\subsection{Results and Analysis}

According to the results of the range analysis on the test results in Table 2, the order of the factors affecting the sorting time is as follows: the material quantity, channel dip angle, and vibration frequency. The optimal level combination is as follows: quantity of material $A$ is 25 , channel dip angle $\alpha$ is $12^{\circ}$, vibration frequency $f$ is $52.5 \mathrm{~Hz}$, and material delivery efficiency is up to $0.51 \mathrm{~s} /$ root.

The vibration frequency had the most significant effect on the material-sorting rate. According to the column of vibration frequency in Table $5, k_{1}<k_{2}$ and $k_{2}>k_{3}$ shows that the material-sorting rate decreases first, then increases with increasing vibration frequency. The main reason is that the increase in vibration frequency causes the material to bounce; thus, the collision occurs, which reduces the sliding speed of the material. However, as vibration frequency continues to increase, the sliding speed of the material increases as well, and the impact 
of the collision is smaller, so the material-sorting rate is improved.

The impact of the material quantity on the materialsorting rate was the second highest factor. According to Table 5, the average time for material-sorting is $1.07 \mathrm{~s} /$ root when the material quantity is 25 . When the material quantities are 20 and 30 , the average time of materialsorting is longer. This is because the vibration effect and sliding speed of the material are the same when vibration frequency is the same. When the quantity of material for sorting and conveying in a single batch is more, the material quantity to be tidied at a single time is also more. However, owing to the material port size, too many materials will cause blockage at the channel mouth, making it difficult for the material to enter the material channel. This results in an increase in time for the material to be sorted. In addition, too small a material quantity will cause the material to be squeezed less in the channel mouth. The materials mainly rely on channel vibration and gravity for entering into the channel, resulting in increased material-sorting time.

The channel dip angle has the least influence on the material-sorting rate. The range $R$ of the three factors in Table 5 shows that the three factors have very little influence on the material-sorting rate. The channel inclination plays a guiding role on the material in the process of material-sorting. The vertical vibration has the effect of letting the material fall and plays an auxiliary role in the material-sorting process.

\section{Conclusions}

Individually feeding disposable blood collection needles during packaging is difficult because of its severely intertwined state while stacking. Based on the physical characteristics of blood collection needles, an automatic feeding device equipped with a compound vibrationtype material-tidying mechanism and a material sorting and conveying mechanism was designed. An orthogonal test analysis was carried out using the experimental prototype.

1. An automatic feeding mode for flexible materials was proposed in this study. The experimental results showed that the automatic feeding mode is feasible.

2. According to the orthogonal test in the compound vibration-type material-tidying and the material sorting and conveying mechanisms, the results demonstrated that the order of factors affecting the total length of blood collection needles after the tidying process were vertical vibration frequency, horizontal vibration amplitude, vertical vibration amplitude, and horizontal vibration frequency. The order of factors affecting the sorting time of blood collection needles were vibration frequency, material quantity, and channel dip angle.

3. The automatic feeding device can realize the feeding efficiency requirement of 80000 roots per day in five channels using the combination of optimum parameters in the compound vibration-type material-tidying and material sorting and conveying mechanisms. This research can provide a reference for the automatic feeding of the large aspect ratio stacked flexible materials that are similar to the blood collection needles.

\section{Authors' Contributions}

CW was in charge of the whole trial; JT and YZ wrote the manuscript; LH and $P C$ assisted with sampling and laboratory analyses. All authors read and approved the final manuscript.

\section{Authors' Information}

Junhua Tong, born in 1984, is currently an associate professor at Faculty of Mechanical Engineering and Automation, Zhejiang Sci-Tech University, China. He received his PhD degree from Zhejiang University, China, in 2014. His research interests include intelligent agricultural equipment and robotics. He has authored over 15 publications in journals and conference proceedings. Yingpeng Zhu, born in 1993, is currently a master candidate at Faculty of Mechanical Engineering and Automation, Zhejiang Sci-Tech University, China. Leiying He, born in 1983, is currently a lecturer at Faculty of Mechanical Engineering and Automation, Zhejiang Sci-Tech University, China. He received his PhD degree from Zhejiang Sci-Tech University, China, in 2014. His research interests include Mechanical design and machine vision systems.

Chuanyu Wu, born in 1976, is currently a professor and a PhD candidate supervisor at Faculty of Mechanical Engineering and Automation, Zhejiang SciTech University, China. His research interests include mechanism design theory and robotics.

Peilin Cheng, born in 1992, he received his master degree from Zhejiang Sci-Tech University, China.

\section{Competing Interests}

The authors declare that they have no competing interests.

\section{Funding}

Supported by National Natural Science Foundation of China (Grant No. 51675488), Public projects of Zhejiang Province (Grant No. LGG18E050020), and Science Foundation of Zhejiang Sci-Tech University (Grant No. $16022015-Y)$.

\section{Author Details}

${ }^{1}$ Faculty of Machinery and Automation, Zhejiang Sci-Tech University, Hangzhou 310018, China. ${ }^{2}$ Key Laboratory of Transplanting Equipment and Technology of Zhejiang Province, Hangzhou 310018, China.

Received: 6 June 2018 Revised: 27 February 2019 Accepted: 8 August 2019

Published online: 20 August 2019

\section{References}

[1] B P Chai, M Y Ma. Application and prospect of automation in packaging machinery. Modern Manufacturing Technology and Equipment, 2017(04): 154-156. (in Chinese) 
[2] H Q Tang, J H Lv. Present situation and development trend of packaging machinery in China. China Packaging Industry, 2015(18): 81-84. (in (hinese)

[3] C J Pang. Application of automation technology in packaging machinery. Electronic Technology \& Software Engineering, 2017(16): 136. (in Chinese)

[4] S C Wang, Z Q Chen, Z K Sun, et al. Mechanism analysis and experiment for automatic egg feeding device. Transactions of the Chinese Society for Agricultural Machinery, 2018, 49(1): 352-357. (in Chinese)

[5] J H Tong, P L Cheng, CY Wu, et al. Automatic sorting and picking manipulator for blood collecting needles in stacking state. Packaging Engineering, 2018(3): 124-129. (in Chinese)

[6] P L Cheng. Research on automatic feeding technology of soft-slender material in stacking state based on compound vibration. Hangzhou: Zhejiang Sci-Tech University, 2018. (in Chinese)

[7] C F Wan, SX Lu. Design of automatic feeding device for cylindrical roller CNC machining. Journal of Hubei Institute of Technology, 2015, 31(3): 10-13. (in Chinese)

[8] Y Atoum, S Srivastava, X Liu. Automatic feeding control for dense aquaculture fish tanks. IEEE Signal Processing Letters, 2015, 22(8): 1089-1093.

[9] Y P Peng, S Gu, Q Chu, et al. Design of stock feeding device of grafting robot for solanaceae. Transactions of the Chinese Society of Agricultural Engineering, 2016, 32(11): 76-82. (in Chinese)

[10] Z C Shang, S F Meng, HYWu, et al. Design of automatic pipe cutting feeding device. Science \& Technology Information, 2012, (31): 74-83. (in Chinese)

[11] Z Ma. Automatic material feeding device for container wood floor. Journal of Shanghai Ship and Shipping Research Institute, 2015, 38(02): 19-22. (in Chinese)

[12] T L Qi, S Q Zhang, H G Wang, et al. Design and mechanics analysis of automatic pulp feeding machine. Machine Tool \& Hydraulics, 2015, 43(16): 35-38. (in Chinese)

[13] J B Chen, D Han, XTWang, et al. Multi-fingered coordinated control for dexterous robotic hand. Journal of Mechanical Engineering, 2014, 50(5): 42-47. (in Chinese)

[14] K Baizid, G Giglio, F Pierri, et al. Experiments on behavioral coordinated control of an Unmanned Aerial Vehicle manipulator system. IEEE International Conference on Robotics and Automation, IEEE, 2015: 4680-4685.

[15] J P Singh, K Lochan, NV Kuznetsov, et al. Coexistence of single- and multi-scroll chaotic orbits in a single-link flexible joint robot manipulator with stable spiral and index-4 spiral repellor types of equilibria. Nonlinear Dynamics, 2017(4): 1-23.

[16] B Jin, LX Lin. Design and force control of an underactuated robotic hand for fruit and vegetable picking. Journal of Mechanical Engineering, 2014, 50(19): 1-8. (in Chinese)

[17] J Zhang. Analysis of adaptive flexibility of three-finger manipulator with six-joint for grasping apple. Transactions of the Chinese Society of Agricultural Engineering, 2010, 26(1): 140-144. (in Chines)

[18] J Zhang, W Li, J Yu, et al. Development of a virtual platform for telepresence control of an underwater manipulator mounted on a submersible vehicle IEEE Transactions on Industrial Electronics, 2017, 64(2): 1716-1727. (in Chinese)

[19] M Ramalingam, G L Samuel. Investigation on the conveying velocity of a linear vibratory feeder while handling bulk-sized small parts. International Journal of Advanced Manufacturing Technology, 2009, 44(3-4): 372-382.

[20] M Loy, G Reinhart. A new modular feeding system and its economic scope of application. Production Engineering, 2010, 4(4): 357-362.

[21] Y B Wang, X G Zhao, L M Xu. Experiment and directional movement technology of corn seed based on electromagnetic vibration. Transactions of the Chinese Society for Agricultural Machinery, 2015, 46(01): 79-88. (in Chinese)

[22] ZX Meng, D F Xiao, H Yin, et al. Mechanism error compensation method of parallel curve feeding platform. Journal of Beijing Forestry University, 2016, 38(09): 95-101. (in Chinese)

[23] L Xu, L Li, X C Zhang, et al. The key technical principle for packing the filamentous materials. Packaging Engineering, 2017, 38(11): 138-142. (in Chinese)

[24] M L Chandravansh, A K Mukhopadhyay. Dynamic analysis of vibratory feeder and their effect on feed particle speed on conveying surface. Measurement, 2017, 101: 145-156.

[25] J J Xing, L M Xu, X D Liu, et al. Simulation and test of corn seeds'dispersion and arraying transport in electromagnetic vibration hopper. Transactions of the Chinese Society of Agricultural Engineering, 2017, 33(13): 32-39. (in Chinese)

[26] Y B Jiang, Y J Wang, G L Lin, et al. An automatic feeding system with a linear piezoelectric actuator, driving circuit and position sensors. Microsystem Technologies, 2018, 24(4): 1909-1917. (in Chinese)

[27] DY Koo, S H Han, S H Lee. An object-oriented configuration design method for paper feeding mechanisms. Expert Systems with Applications, 1998, 14(3): 283-289.

[28] C J Han, W Z Yang, X J Zhang, et al. Design and experiment of automatic feeding system of tray seedlings transplanter. Transactions of the Chinese Society of Agricultural Engineering, 2013, 29(8): 51-61. (in Chinese)

[29] J H Tong, HY Jiang, Z H Jiang, et al. Experiment on parameter optimization of gripper needles clamping seedling plug for automatic transplanter. Transactions of the Chinese Society of Agricultural Engineering, 2014, 30(16): 8-16. (in Chinese)

[30] J H Tong, Q C Yu, J H Geng, et al. Design and experiment of automatic handling and replanting device for double root-cut grafting machine. Journal of Agricultural Machinery, 2017, 48(10): 59-66. (in Chinese)

[31] H Doyo. Compiler: US, 9533844B2. 2017-01-03[2019-02-20]. Paper feeding device and image forming apparatus. IEEE Antennas \&Wireless Propagation Letters, 2017: 497-497. http://www.freepatentsonline.com/9533844.html.

[32] Y J Zhao, Z Ma, Y Chen, et al. Study on design of automatic feeding mechanism of transplanter. Journal of Agricultural Mechanization Research, 2018(7): 68-71. (in Chinese)

\section{Submit your manuscript to a SpringerOpen ${ }^{\circ}$ journal and benefit from:}

- Convenient online submission

- Rigorous peer review

- Open access: articles freely available online

- High visibility within the field

Retaining the copyright to your article

Submit your next manuscript at springeropen.com 